\section{ORIGINAL RESEARCH}

\author{
J.K. Park \\ S.-E. Kim \\ G.S. Trieman \\ D. Parker \\ E.-K. Jeong
}

\title{
High-Resolution Diffusion-Weighted Imaging of Neck Lymph Nodes Using 2D-Single-Shot Interleaved Multiple Inner Volume Imaging Diffusion-Weighted Echo-Planar Imaging at 3T
}

\begin{abstract}
BACKGROUND AND PURPOSE: 2D-sS-IMIV-DWEPI is an SS-DWEPI with greatly reduced geometric distortion. The purposes of this paper are to 1) evaluate of the utility of 2D-ss-IMIV-DWEPI for high-resolution neck LN imaging; 2) determine whether 2D-ss-IMIV-DWEPI can depict normal LN hilum; and 3) evaluate whether the inclusion of LN hilum within ROls affects the measured LN ADC.
\end{abstract}

MATERIALS AND METHODS: HR-DWI was acquired with 1- $\mathrm{mm}^{2}$ in-plane resolution and 2-mm section thickness by using 2D-ss-IMIV-DWEPI. In total, 58 LNs from 18 subjects were evaluated. The ADC map was calculated by using DWI with $b=10$ and $300 \mathrm{~s} / \mathrm{mm}^{2}$. In those LNs where the LN hilum could be recognized, the LN ADC was measured with and without inclusion of the hilum, and the mean difference of the resulting $A D C$ values was determined.

RESULTS: The hilar structure was identified by DWI in 15 LNs. The ADC of the hilum was $1.981 \pm$ $0.331 \times 10^{-3} \mathrm{~mm}^{2} / \mathrm{s}$. In these $15 \mathrm{LNs}$, the ADC value excluding hilar structure was significantly lower than the ADC value including hilar structure $\left(0.983 \pm 0.169\right.$ versus $1.206 \pm 0.244 \times 10^{-3} \mathrm{~mm}^{2} / \mathrm{s} ; P<$ .0001). The mean ADC in a total of $58 \mathrm{LNs}$ excluding the hilar structure was significantly lower than the value obtained including the hilar structure $\left(1.034 \pm 0.183\right.$ versus $1.095 \pm 0.213 \times 10^{-3} \mathrm{~mm}^{2} / \mathrm{s} ; P=$ .0002).

concLUSIONS: HR-DWI of neck LNs obtained by using 2D-ss-IMIV-DWEPI could identify the hilar structure. The ADC of normal neck LNs seemed significantly different when the hilum was included. The results suggest that HR-DWI may be helpful to aid selection of proper ROls within LNs for accurate and reliable ADC measurements.

ABBREVIATIONS: $A D C=$ apparent diffusion coefficient; $\mathrm{DWI}=$ diffusion-weighted imaging; $\mathrm{EPI}=$ echo-planar imaging; $H R=$ high-resolution; $L N=$ lymph node; $P E=$ phase-encoding; $R F=$ radio frequency; $\mathrm{ROI}$ = region of interest; $\mathrm{SNR}$ = signal-to-noise ratio; 2D-Ss-IMIV-DWEPI $=2 \mathrm{D}$-singleshot interleaved multiple inner volume imaging diffusion-weighted echo-planar imaging

$\mathbf{L}^{\mathrm{N}}$ $\mathrm{N}$ metastasis is one of the most important prognostic factors in patients with head and neck squamous cell carcinoma. One of the major goals of diagnostic imaging in these patients is accurate prediction of LN metastasis not only for the planning of appropriate treatment but also for monitoring the treatment response. Conventional criteria used in clinical imaging studies are morphologic criteria including the maximum short axial diameter, presence of necrosis, loss of LN hilum, heterogeneous enhancement, and perinodal infiltration. A size criterion and presence of necrosis are relatively objective, but the other criteria are less objective and depen-

\section{Received July 10, 2010; accepted after revision November 26}

From the Utah Center for Advanced Imaging Research (J.K.P., S.-E.K., D.P., E.-K.J), Department of Radiology (S.-E.K., D.P., E.-K.J.), and Department of Surgery (G.S.T.), University of Utah, Salt Lake City, Utah; Department of Radiology (J.K.P.), Jeju National University Hospital, Jeju, South Korea; and Department of Veterans Affairs (G.S.T.), VASLCHCS, Salt Lake City, Utah.

This work was supported by National Institutes of Health grants R01 HL 48223, R01 HL 57990, R21 NS052424, and R21 EB005705; and Clinical Merit Review Grant from the Veterans Administration Health Care System, Siemens Medical Solutions, the Mark H. Huntsman Endowed Chair, and the Margolis Foundation.

Please address correspondence to Eun-Kee Jeong, PhD, University of Utah, Department of Radiology, Utah Center for Advanced Imaging Research, 729 Arapeen Dr, Salt Lake City, UT 84108; e-mail: ekj@ucair.med.utah.edu

DOI 10.3174/ajnr.A2539

Indicates open access to non-subscribers at www.ajnr.org dent on the interpretation of the radiologist. To improve the accuracy of detection, new MR imaging contrast agents such as ultrasmall particles of iron oxide have been developed and show promising results. ${ }^{1,2}$ However, the contrast agent requires repeated MR imaging scans and has associated risks. ${ }^{2}$ As MR imaging hardware such as gradient systems and multichannel radiofrequency coils have been improved, ss DWEPI has been applied to head and neck imaging. DWEPI provides a quantitative measurement of the ADC of water protons in tissue. According to recent reports, an ADC value that is significantly lower in the metastatic LNs than that in the benign LNs can be used to differentiate malignant LNs from benign $\mathrm{LNs}^{3-6}$ and can be a marker for prediction and early detection of chemoradiation therapy response. ${ }^{7,8}$ DWEPI also can be used for the early detection of recurrence. ${ }^{9}$ However, DWEPI has some drawbacks due to its EPI acquisition, leading to limited spatial resolution and poor image quality. EPI has intrinsically low bandwidth along the PE dimension, and any field inhomogeneity-caused frequency offset causes a phase error that progressively disperses along the $\mathrm{k}_{\mathrm{y}}$ dimension in the $\mathrm{ab}-$ sence of refocusing pulses. This long echo train makes EPI susceptible to geometric distortion caused by magnetic susceptibility and blurring caused by $\mathrm{T} 2{ }^{*}$ decay. Geometric distortion and blurring in EPI is inversely proportional to the increase of PE steps and PE FOV. For HR-DWEPI, PE steps 


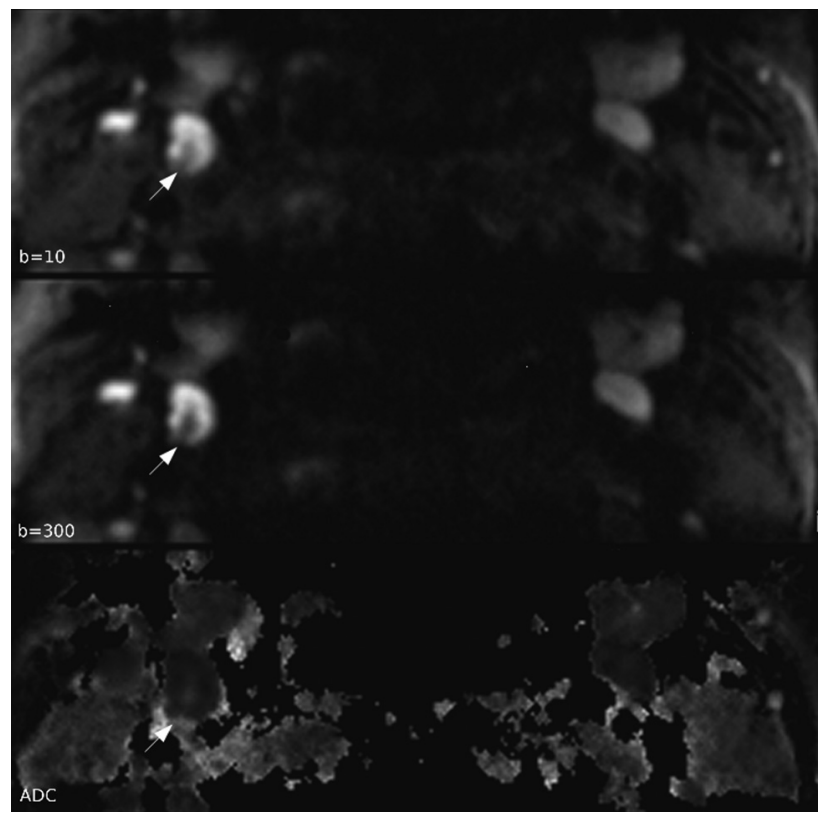

Fig 1. Typical DW images and $A D C$ map. Normal $L N$ hilum is seen as a central linear structure with low signal intensity on $b=10$ and $b=300$ images and high signal intensity on $\mathrm{ADC}$ map (white arrow) in the right side level $2 \mathrm{LN}$ with a 6.8-mm diameter. ADC of hilum is $2.152 \times 10^{-3} \mathrm{~mm}^{2} / \mathrm{s}$, which is close to the diffusivity of bulk water.

must be increased. Thus, it is difficult to obtain DWI with sufficient high spatial resolution to evaluate fine detail of small LNs with $<10-\mathrm{mm}$ axial diameter. The typical axial spatial resolution of DWEPI used in head and neck imaging has been $2 \times 2 \mathrm{~mm}$ with $4-5 \mathrm{~mm}$ thickness. ${ }^{3,5,6,9}$

The conventional 2D-DWEPI technique, which is most widely used for DWI, is limited to intracranial DWI applications because of the geometric distortion that occurs in other locations. The geometric distortion and blurring in DWEPI can be reduced by reducing the imaging FOV in the PE direction, which induces aliasing-artifact in the PE direction. To reduce $P E$ FOV without aliasing-artifact, we have recently developed IMIV imaging. ${ }^{10-12}$ When combined with DWEPI, the resulting technique, 2D-ss-IMIV-DWEPI, can perform a HR multisection DWEPI with reduced geometric distortion and blurring. In a normal LN, the distribution of lymphoid cells and lymph fluid may be different from the cortex to the hilum. The LN hilum consists of the convergence of the medullary sinus, efferent lymphatic vessels, arteries, veins, and fatty tissue, and the hilum includes a collection of lymph fluid from the LN cortex and medulla. ${ }^{13,14}$ There is a possibility that the ADC may vary according to the location within the LN. To our knowledge, no previous DWI studies presented the ADC value of the LN hilum and its effects on the measurement of ADC. There was only one previous report describing that ADC of the central part of the LNs was higher than that of the other parts of LNs. ${ }^{15}$ The purposes of this paper are to 1) evaluate of the utility of 2D-ss-IMIV-DWEPI for HR neck LN imaging; 2) determine whether 2D-ss-IMIV-DWEPI can depict normal LN hilum; and 3) evaluate whether the inclusion of LN hilum within ROIs affects the measured LN ADC.

\section{Materials and Methods}

This study was approved by the University of Utah Institutional Review Board committee, and all patients gave written informed con- sent before being included. From July 2009 to April 2010, 18 patients (age range, 48-81 years) scheduled for carotid endarterectomy were consecutively enrolled. None of the enrolled subjects had any head and neck malignancy.

\section{Imaging Acquisition}

DW images were acquired for the evaluation of carotid atherosclerosis by using 2D-ss-IMVI-DWEPI. All studies were performed on a Siemens Trio 3T MR imaging scanner (Siemens, Erlangen, Germany). Detail on the MR imaging data acquisition techniques has been published previously. ${ }^{10-12,16}$ Only aspects particular to this study are presented here. The center section was positioned near the carotid bifurcation in all studies. A home-made bilateral dual-element phased array surface RF coil designed for neck imaging was used. The DWI imaging parameters were receiver bandwidth $=1.086 \mathrm{kHz} /$ pixel, $\mathrm{FOV}=160 \times 40 \mathrm{~mm}^{2}$, imaging matrix $=160 \times 40,2$-mm section thickness, effective TE $=56 \mathrm{~ms}$, TR $=3$ seconds, echo-train length of 40 per each section, 32 averages (magnitude), and interleaved acquisition of $12-16$ contiguous sections. The in-plane spatial resolution for a typical data acquisition was $1 \times 1 \mathrm{~mm}^{2}$ with display resolution $0.5 \times 0.5 \mathrm{~mm}^{2}$, after zero-filled interpolation. DW images were acquire with $b=10 \mathrm{~s} / \mathrm{mm}^{2}$ and with $b=300 \mathrm{~s} / \mathrm{mm}^{2}$ along the section selection direction with imaging time 3 minutes 20 seconds.

\section{Data Analysis}

A neuroradiologist reviewed all the imaging studies. LNs that had a maximum short axial diameter $>4 \mathrm{~mm}$ on $b$-10 images were included. ADC maps (square millimeters per second) were measured in a total of 58 LNs by using in-house software developed in IDL (ITT Visual Information Systems, Boulder, Colorado). For ADC measurement, ROIs were placed in the LNs, which were identified by the typical high signal intensity on the $b=10$ and $b=300$ images and low signal intensity on the ADC map. The ROIs were placed over the entire LN. In the cases in which DWI revealed the LN hilum clearly, we measured the ADC in the hilum, and the LN ADC was measured twice by including or excluding the hilar structures. SNR was calculated from the equation $\mathrm{SNR}_{\mathrm{LN}}=$ signal intensity ${ }_{\mathrm{LN}} / \mathrm{SD}_{\text {noise }}$, where signal intensity $\mathrm{LN}_{\mathrm{LN}}$ is the mean signal intensity of ROIs at LNs and $\mathrm{SD}_{\text {noise }}$ is the standard deviation of background signal intensity.

\section{Statistical Analyses}

The Kolmogorov-Smirnov test was conducted by using SPSS, version 15.0 (SPSS, Chicago, Illinois) to diagnose normality of data distribution. ADC values and LN sizes were normally distributed. In the cases in which DWI revealed hilar structure, a paired samples $t$ test was performed to assess the difference of the ADC between ROIs including and excluding the hilum structure. In addition, a paired samples $t$ test was performed to find the difference of the ADC in a total of 58 LNs according to hilar inclusion in the LNs with the clearly visualized hilum on DWI. The $P$ value was considered significant if $\leq .05$ at confidence interval 95\%.

\section{Results}

None of the DWI showed significant geometric distortion, ghosting, or aliasing artifacts that may hinder ADC measurement. The average LN size was $5.1 \pm 1.1 \mathrm{~mm}$ and maximum size was $7.6 \mathrm{~mm}$. SNR of $b=10$ and $b=300$ images was 129.95 and 93.69 , respectively. In $15 \mathrm{LNs}$, the hilar structure was revealed by DWI (Fig 1). Hilar structure was identified by its typical central location and linear shape with low signal inten- 

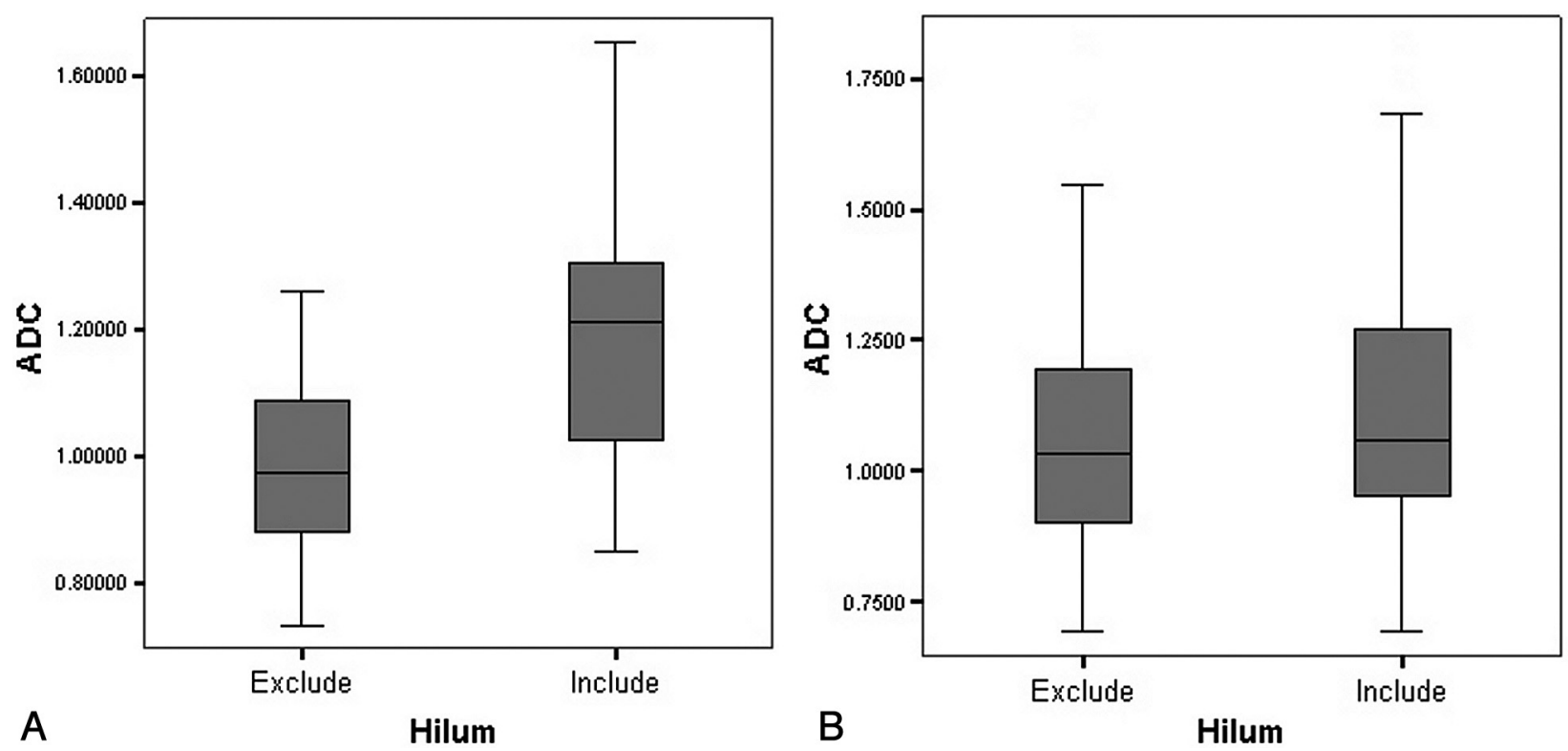

Fig 2. There is significant $A D C$ difference in the $15 \mathrm{LNs}$ in that hilum is identified by DWI and ADC map (A). ADC from ROls excluding hilum is significantly lower than $A D C$ from ROIs including hilum. The variation of ADC is smaller when the hilum was excluded within ROls. Also, there is significant difference in ADC values measured on a total of 58 LNs according to the hilar inclusion $(B)$. ADC is significantly lower in $L$ Ns with ROls in which the hilum is excluded.

\begin{tabular}{|c|c|c|c|c|c|c|}
\hline \multicolumn{7}{|c|}{ Results of ADC values according to hilum inclusion } \\
\hline & \multicolumn{3}{|c|}{ LNs with Visible Hilum $(n=15)$} & \multicolumn{3}{|c|}{ Total LNs $(n=58)$} \\
\hline & Hilum(-) & Hilum(+) & $P$ & Hilum(-) & Hilum(+) & $P$ \\
\hline$A D C$ & $0.983 \pm 0.169$ & $1.206 \pm 0.244$ & $<.0001$ & $1.034 \pm 0.183$ & $1.095 \pm 0.213$ & .0002 \\
\hline
\end{tabular}

Note:-Hilum(-) means that the hilum was excluded within the ROls. Hilum(+) means that the hilum was included within the ROIs.

sity on $b=10$ and $b=300$ images and high signal intensity on the ADC map (Fig 1). ADC of the hilum was $1.981 \pm 0.331 \times$ $10^{-3} \mathrm{~mm}^{2} / \mathrm{s}$. In these $15 \mathrm{LNs}$, the ADC value excluding the hilar structure $\left(0.983 \pm 0.169 \times 10^{-3} \mathrm{~mm}^{2} / \mathrm{s}\right)$ was significantly lower than the ADC value including the hilar structure $\left(1.206 \pm 0.244 \times 10^{-3} \mathrm{~mm}^{2} / \mathrm{s}\right.$ ) at $P<.0001$ (Fig $2 A$ and Table). The mean ADC in a total of $58 \mathrm{LNs}$ obtained by using the ADC excluding hilar structure from 15 LNs (1.034 \pm $0.183 \times 10^{-3} \mathrm{~mm}^{2} / \mathrm{s}$ ) was significantly lower than the ADC in a total of $58 \mathrm{LNs}$ obtained by using the ADC including the hilar structure from $15 \mathrm{LNs}\left(1.095 \pm 0.213 \times 10^{-3} \mathrm{~mm}^{2} / \mathrm{s}\right)$ at $P=$ .0002 (Fig $2 B$ and Table).

\section{Discussion}

In this study, HR-DWI of LNs in the neck could be obtained with acceptable geometric distortion and scanning time by using 2D-ss-IMIV-DWEPI in a 3T MR imaging system. Using HR-DWI, we were able to depict the subcentimetric LNs with short axial dimension of 4-7.6 $\mathrm{mm}$ and reveal hilar structure of these LNs in 15 (25.9\%) of 58 LNs. Neck LN DWI has been usually performed by $1.5 \mathrm{~T} \mathrm{MR}$ imaging system, and there is only 1 report of $3 \mathrm{~T}$ MR imaging. In those studies, the mean $\mathrm{ADC}$ of normal or benign LNs was $1.286 \pm 0.2 \times 10^{-3} \mathrm{~mm}^{2} / \mathrm{s}$, with a range from 1.16 to 1.64 at $1.5 \mathrm{~T},{ }^{3-6,17}$ and $1.057 \pm$ $0.101 \times 10^{-3} \mathrm{~mm}^{2} / \mathrm{s}$ at $3 \mathrm{~T} .{ }^{18}$ In our study, ADC showed variable values depending on the hilar inclusion, with a range from 0.983 to $1.206 \times 10^{-3} \mathrm{~mm}^{2} / \mathrm{s}$. Our ADC values and previous 1.5T MR imaging results showed a wide range of variation of ADC in normal or benign LNs. Our results suggest that this large variation resulted from the variable amount of the LN hilum that was included in the ROIs used for ADC calculation. In our study, we found that LN ROI selection had a significant effect on the measured ADC. One of the morphologic characteristics in normal and benign lymphadenopathy is preservation of LN hilum. In the clinical imaging studies such as sonography, CT, and MR imaging, one can observe variation of hilum size within individual LNs. The volume fraction of hilum compared with total volume of the LNs is usually very small. However, the ADC of the LN hilum was prominently higher than the mean ADC of normal LNs in our study. Therefore, although only a small volume of hilum is included within the LN ROIs, the effect of hilar inclusion on the mean LN ADC may be significant. Our results suggest that more accurate and reliable ADC measurement may be made in the LNs by using HR-DWI.

The previously reported mean ADC of metastatic LNs from head and neck squamous carcinomas was $0.936 \pm 0.1365 \times$ $10^{-3} \mathrm{~mm}^{2} / \mathrm{s}$, with a range from 0.78 to 1.11 in $1.5 \mathrm{~T} \mathrm{MR}$ imaging. ${ }^{3-6,9,19}$ Although lower ADC values were reported at $3 \mathrm{~T}$ than at $1.5 \mathrm{~T}$ in the brain ${ }^{20}$ and in abdominal organs, ${ }^{21}$ there was no significant statistical difference for different field strength in neck imaging. ${ }^{7}$ The water diffusivity should not depend on the magnetic field strength, because the molecular motion is purely a thermal phenomenon, not a nuclear MR characteristic. Although we did not compare the ADC between normal LNs and metastatic LNs, the ADC value that was measured from 15 normal LNs with ROIs excluding the hilum $\left(0.983 \pm 0.1685 \times 10^{-3} \mathrm{~mm}^{2} / \mathrm{s}\right)$ was similar to that of metastatic LNs at $1.5 \mathrm{~T}$ MR imaging. ADC value from LNs with ROIs including hilum was significantly higher than the ADC 
of metastatic LNs at 1.5T MR imaging. Previous reports suggested that the significantly lower ADC in the metastatic LNs was due to the different diffusion environment of water protons in the metastatic LNs, including decreased extracellular space, increased cellularity, and higher nuclear-to-cytoplasmic ratio. However, the higher ADC in the normal or benign LNs may be due to drawing of ROIs including the hilar structure. The higher ADC in the normal or benign LNs may reflect the preservation of LN hilum, one morphologic characteristic of normal or benign LNs. Therefore, the comparison study of ADC between benign and metastatic LNs by using HR-DWI and choosing proper ROIs may be needed to determine the added value of the ADC to differentiate metastatic LNs from benign LNs.

In this study, the PE FOV was not large enough to cover the major neck LN groups. To apply 2D-ss-IMIV-DWEPI for clinical practice, the PE FOV must be increased. However, the geometric distortion will be increased with increased imaging FOV in the PE direction. The IMIV technique can be combined with parallel imaging to increase the imaging FOV in the $\mathrm{PE}$ direction while limiting the geometric distortion. This combination will allow doubling the PE FOV (from 4 to $8 \mathrm{~cm}$ ) in HR-DWI and the same degree of geometric distortion as the DWI protocol used in this study. The b-value used in this study was 300 seconds $/ \mathrm{mm}^{2}$, which is low compared with the typical b-value used in brain DWI. This lower b-value was used because of the possibility that the pulsatile motion due to the arterial blood in the carotid arteries may displace the blood signal intensity on the LN. The effect of perfusion on ADC measurements will increase with the low b-value DWI, and this may result in an artificial increase in calculated ADC. Previous reports confirmed that $\mathrm{b}$-values $>100 \mathrm{~s} / \mathrm{mm}^{2}$ make the perfusion effect on ADC negligible. ${ }^{22,23}$ Our ADC result from a total of 58 LNs was similar to previous 3T MR imaging results with b-values of 0 and $800 \mathrm{~s} / \mathrm{mm}^{2} .^{18}$ For accurate calculation of diffusion matrices, SNR should be maintained within a sufficient range. ${ }^{24} \mathrm{Neck}$ DWEPI is prone to be more sensitive to magnetic susceptibility due to the proximity of tissue with different magnetic susceptibility such as air, bone, and soft tissue. A b-value of $300 \mathrm{~s} / \mathrm{mm}^{2}$ may be considered to be sufficient to produce DWI with high SNR for accurate ADC measurement. A dedicated surface RF coil was used in this study for high SNR. Use of lower performance coils such as those used for clinical MR imaging of the neck may significantly reduce the SNR of the DWI.

One limitation of our study was limited visualization of the LN hilum in the 2D-ss-IMIV-DWEPI images because of the low-resolution nature of DWI. In our study, the LN hilum was identified only in $25.9 \%$ of total LNs. In previous reports, the visualization rate of the hilum in the benign LNs varied with the different imaging modalities. HR CT $(0.25-0.5-\mathrm{mm}$ inplane resolution) and anatomic MR imaging $(0.5-\mathrm{mm}$ inplane resolution) revealed the hilum of the benign LNs in $29 \%$ and $54 \%$ of the examined LNs, respectively. ${ }^{14,25}$ HR sonography revealed the hilum of the benign LNs in the various range from $41 \%$ to $100 \%$ according to the level of the neck LNs, age, and sex. ${ }^{26}$ An alternative way to overcome this shortfall is to use other HR images such as T2 weighted images, which may show the LN hilum, to define the ROI and use these coordinates to measure the $\mathrm{ADC}$ value.

\section{Conclusions}

These results demonstrated that 2D-ss-IMIV-DWEPI can be used for HR-DWI of neck LNs with depiction of the hilar structure of subcentimetric LNs in $25.9 \%$. ADC of normal LNs substantially increased when the LN hilum was included within the selected ROI. HR-DWI may be helpful to aid in proper selection of the ROI within LNs for accurate and reliable ADC measurement.

Disclosures: Dennis L. Parker, Research Support (including provision of equipment or materials): NIH, Siemens, Details: Our work is primarily funded by several NIH grants. We also have a small grant from Siemens Medical Solutions that provides support for a small number of students and research associates to work on research of interest to them.

\section{References}

1. Sigal R, Vogl T, Casselman J, et al. Lymph node metastases from head and neck squamous cell carcinoma: MR imaging with ultrasmall superparamagnetic iron oxide particles (Sinerem MR)-results of a phase-III multicenter clinical trial. Eur Radiol 2002;12:1104-13

2. Anzai Y, Piccoli CW, Outwater EK, et al. Evaluation of neck and body metastases to nodes with ferumoxtran 10-enhanced MR imaging: phase III safety and efficacy study. Radiology 2003;228:777-88

3. Abdel Razek AA, Soliman NY, Elkhamary S, et al. Role of diffusion-weighted MR imaging in cervical lymphadenopathy. Eur Radiol 2006;16:1468-77

4. de Bondt RB, Hoeberigs MC, Nelemans PJ, et al. Diagnostic accuracy and additional value of diffusion-weighted imaging for discrimination of malignant cervical lymph nodes in head and neck squamous cell carcinoma. Neuroradiology 2009;51:183-92

5. Holzapfel K, Duetsch S, Fauser C, et al. Value of diffusion-weighted MR imaging in the differentiation between benign and malignant cervical lymph nodes. Eur J Radiol 2009;72:381-87

6. Vandecaveye V, De Keyzer F, Vander Poorten V, et al. Head and neck squamous cell carcinoma: value of diffusion-weighted MR imaging for nodal staging. Radiology 2009;251:134-46

7. Kim S, Loevner L, Quon H, et al. Diffusion-weighted magnetic resonance imaging for predicting and detecting early response to chemoradiation therapy of squamous cell carcinomas of the head and neck. Clin Cancer Res 2009;15:986-94

8. Kato H, Kanematsu M, Tanaka O, et al. Head and neck squamous cell carcinoma: usefulness of diffusion-weighted MR imaging in the prediction of a neoadjuvant therapeutic effect. Eur Radiol 2009;19:103-09

9. Vandecaveye V, De Keyzer F, Nuyts S, et al. Detection of head and neck squamous cell carcinoma with diffusion weighted MRI after (chemo)radiotherapy: correlation between radiologic and histopathologic findings. Int J Radiat Oncol Biol Phys 2007;67:960-71

10. Jeong EK, Kim SE, Guo J, et al. High-resolution DTI with 2D interleaved multislice reduced FOV single-shot diffusion-weighted EPI (2D ss-rFOVDWEPI). Magn Reson Med 2005;54:1575-79

11. Jeong EK, Kim SE, Kholmovski EG, et al. High-resolution DTI of a localized volume using 3D single-shot diffusion-weighted stimulated echo-planar imaging (3D ss-DWSTEPI). Magn Reson Med 2006;56:1173-81

12. Kim SE, Jeong EK, Shi XF, et al. Diffusion-weighted imaging of human carotid artery using 2D single-shot interleaved multislice inner volume diffusionweighted echo planar imaging (2D ss-IMIV-DWEPI) at 3T: diffusion measurement in atherosclerotic plaque. J Magn Reson Imaging 2009;30:1068-77

13. Rubaltelli L, Proto E, Salmaso R, et al. Sonography of abnormal lymph nodes in vitro: correlation of sonographic and histologic findings. AJR Am J Roentgenol 1990; $155: 1241-44$

14. Uematsu T, Sano M, Homma K. In vitro high-resolution helical CT of small axillary lymph nodes in patients with breast cancer: correlation of CT and histology. AJR Am J Roentgenol 2001;176:1069-74

15. Xue HD, Li S, Sun HY, et al. Experimental study of inflammatory and metastatic lymph nodes with diffusion weighted imaging on animal model: comparison with conventional methods. Chin Med Sci J 2008;23:166-71

16. Kim TH, Zollinger L, Shi XF, et al. Quantification of diffusivities of the human cervical spinal cord using a 2D single-shot interleaved multisection inner volume diffusion-weighted echo-planar imaging technique. AJNR Am J Neuroradiol 2010;31:682-87

17. Kwee TC, Takahara T, Luijten PR, et al. ADC measurements of lymph nodes: inter- and intra-observer reproducibility study and an overview of the literature. Eur J Radiol 2010;75:215-20

18. Srinivasan A, Dvorak R, Rohrer S, et al. Initial experience of 3-Tesla apparent diffusion coefficient values in characterizing squamous cell carcinomas of the head and neck. Acta Radiol 2008;49:1079-84

19. King AD, Ahuja AT, Yeung DK, et al. Malignant cervical lymphadenopathy: diagnostic accuracy of diffusion-weighted MR imaging. Radiology 2007;245:806-13 
20. Huisman TA, Loenneker T, Barta G, et al. Quantitative diffusion tensor MR imaging of the brain: field strength related variance of apparent diffusion coefficient (ADC) and fractional anisotropy (FA) scalars. Eur Radiol 2006;16:1651-58

21. Dale BM, Braithwaite AC, Boll DT, et al. Field strength and diffusion encoding technique affect the apparent diffusion coefficient measurements in diffusion-weighted imaging of the abdomen. Invest Radiol 2010;45:104-08

22. Le Bihan D, Breton E, Lallemand D, et al. Separation of diffusion and perfusion in intravoxel incoherent motion MR imaging. Radiology 1988; 168:497-505
23. Yeung DK, Wong SY, Griffith JF, et al. Bone marrow diffusion in osteoporosis: evaluation with quantitative MR diffusion imaging. J Magn Reson Imaging 2004;19:222-28

24. Jones DK, Basser PJ. "Squashing peanuts and smashing pumpkins": how noise distorts diffusion-weighted MR data. Magn Reson Med 2004; 52:979-93

25. Sumi M, Van Cauteren M, Nakamura T. MR microimaging of benign and malignant nodes in the neck. AJR Am J Roentgenol 2006;186:749-57

26. Ying $M$, Ahuja A, Brook F. Sonographic appearances of cervical lymph nodes: variations by age and sex. J Clin Ultrasound 2002;30:1-11 